\title{
Robótica Educacional como Método Principal de Ensino em Práticas de Programação
}

\author{
Rachel C. D. Reis ${ }^{1}$, Larissa F. Rodrigues ${ }^{1}$, Pablo L. A. Munhoz ${ }^{1}$, Kamila T. Lyra ${ }^{2}$, \\ Seiji Isotani \\ ${ }^{1}$ Universidade Federal de Viçosa, Campus Rio Paranaíba \\ ${ }^{2}$ Universidade de São Paulo, Campus São Carlos \\ rachel.reis@ufv.br, larissa.f.rodrigues@ufv.br, pablo.munhoz@ufv.br, \\ kalyra_03@usp.br, sisotani@icmc.usp.br
}

\begin{abstract}
The use of Educational Robotic to support programming teaching has been investigated by several studies in the literature. Despite of contributions, we observed the lack of studies that use Arduino as main teaching method in programming disciplines. Therefore, this paper aims at evaluating students' motivation when they use Arduino as a teaching method in the practical classes to present the programming initial concepts. An experimental study, carried out with 54, undergraduates showed that students' motivation in the perception choice subscale was significantly higher in the Arduino teaching method compared to the Traditional teaching method.
\end{abstract}

Resumo. $O$ uso da Robótica Educacional para apoiar o ensino de programação tem sido investigado por diversos trabalhos na literatura. Apesar das contribuições, observou-se a carência de estudos que utilizem o Arduino como método principal de ensino em disciplinas de programação. Logo, este artigo tem como objetivo avaliar a motivação dos alunos ao utilizar o Arduino como método de ensino nas aulas práticas para apresentação dos conceitos iniciais de programação. Um estudo experimental, realizado com 54 alunos de graduação, mostrou que a motivação dos alunos na subescala percepção de escolha foi significativamente maior no método de ensino Arduino comparado ao método de ensino Tradicional.

\section{Introdução}

O alto índice de reprovação e evasão escolar em disciplinas de Introdução a Programação tem sido cada vez mais comum nos cursos superiores da área de exatas, em especial para os cursos de Tecnologia da Informação (TI), por exemplo, Sistemas de Informação e Ciência da Computação [Bosse e Gerosa 2015]. Conseguir a motivação dos alunos é um dos grandes desafios no que se refere ao ensino de disciplinas de programação [Piva Jr. e Freitas 2010]. O uso da metodologia tradicional, na qual o aluno é remetido primeiramente à teoria e depois à resolução prática de exercícios no computador, não tem despertado o interesse dos alunos. Nesse sentido, pesquisadores ressaltam a importância de utilizar outras práticas e técnicas de ensino-aprendizagem nas disciplinas de programação [Queiroz e Rebouças 2018]. 
A Robótica Educacional tem sido utilizada por diversos trabalhos na literatura como técnica de ensino-aprendizagem lúdica que desenvolve no aluno a capacidade de pensar e resolver problemas [Santos et al. 2018]. Essa técnica baseia-se em ambientes de aprendizagem que utilizam kits de montagem compostos por diversas peças como LEDs, sensores e motores controlados por aplicações computacionais. Nesse contexto, tem-se o Arduino que é formado por uma placa eletrônica que pode facilmente ser conectada a um computador e programada por meio de uma interface de desenvolvimento [Banzi e Evans 2014].

Apesar da existência de pesquisas sobre o uso do Arduino na área de ensino de lógica e programação [Santos et al. 2018], não foram encontrados na literatura trabalhos que investigassem sua influência na motivação dos alunos, quando utilizado como método principal de ensino. Logo, este artigo tem como objetivo analisar a motivação dos alunos ao utilizar o Arduino como método principal de ensino nas aulas práticas da disciplina de Programação. Essa análise será realizada para os primeiros conceitos vistos na disciplina: estruturas de decisão, estruturas de repetição, vetor/matriz.

Além dessa seção introdutória, este trabalho está organizado da seguinte forma: na Seção 2 são apresentados os trabalhos relacionados. Na sequência, a Seção 3 descreve o estudo experimental. Na Seção 4 são mostrados e discutidos os resultados e, finalmente, na Seção 5 são apresentadas as conclusões.

\section{Trabalhos Relacionados}

As pesquisas sobre o uso da Robótica Educacional (utilizando o Arduino) como estratégia para desenvolvimento do raciocínio lógico e ensino dos conceitos de programação não é muito ampla [Silva et al. 2016]. No ensino superior ainda é mais escasso. Neste contexto, são apresentados nesta seção os trabalhos de Stefanello et al. (2013) e Cardoso e Antonello (2015).

Stefanello et al. (2013) desenvolveram um estudo com 44 alunos de um curso superior em Sistemas de Informação ( 32 do $1^{\circ}$ período e 12 do $8^{\circ}$ período) para avaliar os benefícios da robótica educacional nas disciplinas de programação. O estudo foi realizado em três etapas: palestras sobre robótica (utilizando Arduino) e sua relação com a programação, minicurso com foco em robótica e aplicação de questionário de satisfação. Os resultados dos questionários mostraram que a robótica contribuiu para o desenvolvimento do raciocínio lógico dos alunos para resolução de problemas.

Cardoso e Antonello (2015) planejaram o oferecimento de duas disciplinas (Algoritmo e Oficina de Criatividade), do primeiro semestre do curso de Sistemas de Informação, para melhorar o aprendizado dos conceitos básicos de algoritmo. As práticas das disciplinas envolveram quatro atividades: (1) dinâmicas de grupo, para integração dos alunos; (2) programação visual por blocos, para criação de programas utilizando blocos funcionais; (3) uso do Visualg, para desenvolvimento de pseudocódigos; e (4) kits de Arduino (somente na disciplina de Oficina de Criatividade), para aplicação de conceitos abstratos de programação (estruturas de decisão e repetição). Os resultados, referente ao relato de 46 alunos, mostraram que o Arduino contribuiu para a melhor compreensão e aprendizado dos conceitos de algoritmo.

No intuito de contribuir para a ampliação das pesquisas em Robótica Educacional no âmbito do ensino superior, este trabalho se propõe a avaliar a motivação 
VIII Congresso Brasileiro de Informática na Educação (CBIE 2019)

Anais do XXV Workshop de Informática na Escola (WIE 2019)

dos alunos ao utilizar o Arduino como método principal de ensino nas aulas práticas de Programação. Essa avaliação será feita por meio de um estudo experimental realizado no $1^{\circ}$ semestre de 2019.

\section{Estudo Experimental}

Este trabalho foi desenvolvido com duas turmas da disciplina de Programação (T1 e T2) da Universidade Federal de Viçosa, campus Rio Paranaíba (UFV-CRP), totalizando 54 alunos. Desse total 68,5\% são alunos repetentes, ou seja, já haviam cursado a disciplina de Programação pelo menos uma vez e 31,5\% são alunos ingressantes, ou seja, estavam cursando a disciplina pela primeira vez.

A turma T1 foi definida como grupo controle, ou seja, baseou-se no método de ensino Tradicional (editor + lista) que consiste na resolução de listas de exercícios sem contextualização utilizando um editor de programação (ex.: Code Blocks). Por outro lado, a turma T2 baseou-se no método de ensino com Arduino (editor + circuito + lista) que define um conjunto de exercícios práticos contextualizados ${ }^{1}$ que permite ao aluno visualizar na prática (circuito Arduino) a execução do seu código (ex.: piscar LEDs). Ambos os métodos de ensino utilizaram a linguagem de programação C.

Este estudo foi realizado em três etapas nas aulas práticas da disciplina de Programação. Vale ressaltar que as aulas práticas fazem parte do programa analítico da disciplina de Programação oferecida pelo curso de Sistemas de Informação da UFVCRP com carga horária de 2 horas/aula por semana. Logo, as aulas práticas das turmas $\mathrm{T} 1$ e T2 foram ministradas de forma independente uma vez por semana.

Na primeira etapa (semana 1), a professora explicou o método de ensino a ser utilizado por cada turma: T1 (Tradicional) e T2 (Arduino) e fez uma demonstração sobre a aplicação do método para resolução de um exercício. Na sequência, os alunos foram orientados a resolver um exercício na prática usando os instrumentos especificados para cada método de ensino: Arduino (editor Arduino + circuito + lista), Tradicional (editor Code Blocks + lista).

A segunda etapa foi realizada em três aulas práticas (semanas 2, 3 e 4) de 2 horas/aula cada. Em cada aula, os alunos trabalharam na resolução de uma lista de exercícios referente a um conceito, ou seja, na semana 2 foi trabalhado o conceito de estrutura de decisão; na semana 3, o conceito de estrutura de repetição; e na semana 4, o conceito de vetor/matriz.

Por fim, na terceira etapa (semana 5) as turmas T1 e T2 responderam ao questionário Intrinsic Motivation Inventory (IMI) [Deci e Ryan 1985], versão traduzida para Português do Brasil [Pedro, 2016], avaliando sua motivação em relação às aulas práticas utilizando, respectivamente, o método Tradicional e Arduino. O questionário IMI avalia a motivação de um indivíduo enquanto resolve uma atividade em quatro subescalas: interesse e aproveitamento, percepção de competência, percepção de escolha e pressão e tensão.

\footnotetext{
${ }^{1}$ Um exercício contextualizado descreve uma situação-problema relacionada ao dia-a-dia do aluno (ex.: cotidiano, Universidade) com o intuito de instigar o raciocínio lógico dos alunos [Freitas et al., 2014].
} 
VIII Congresso Brasileiro de Informática na Educação (CBIE 2019)

Anais do XXV Workshop de Informática na Escola (WIE 2019)

\section{Resultados e Discussão}

Este estudo é guiado pela seguinte questão de pesquisa QP: “A motivação dos alunos ao utilizar o método Arduino nas aulas práticas da disciplina de Programação é maior do que utilizando o método Tradicional?".

No intuito de responder à questão de pesquisa acima, foram formuladas quatro hipóteses distintas, uma para cada subescala da motivação. A hipótese alternativa $\left(\mathrm{H}_{1}\right)$ supõe que os valores obtidos da motivação para as subescalas da motivação interesse $e$ aproveitamento, percepção de escolha, percepção de competência e pressão e tensão sejam significativamente diferentes entre o uso do método Arduino e método Tradicional. Por outro lado, a hipótese nula $\left(\mathrm{H}_{0}\right)$ supõe que não existe diferença significativa entre os métodos Tradicional e Arduino.

Para análise dos dados, obtidos a partir do estudo experimental apresentado na Seção 3, foi executado o teste- $t$ com intervalo de confiança de $95 \%$ para comparar a motivação da turma T1 que utilizou o método de ensino Tradicional e a turma T2 que utilizou o método de ensino com Arduino.

De acordo com o resultado do teste-t, a média da motivação dos alunos na subescala percepção de escolha no método Arduino $(\mu=5,27)$ foi significativamente maior $(p<, 05)$ que a média da motivação no método Tradicional $(\mu=4,37)$. Isso evidencia que houve maior aceitação dos alunos pelo método Arduino do que pelo método Tradicional. Logo, a hipótese nula $\left(\mathrm{H}_{0}\right)$ para essa subescala é negada, ou seja, existe diferença significativa entre os métodos de ensino e, nesse caso, essa diferença foi a favor do método Arduino. Com relação às outras subescalas (interesse $e$ aproveitamento, percepção de competência, tensão e pressão) a hipótese nula $\left(\mathrm{H}_{0}\right)$ é aceita, ou seja, não houve diferença significativa na motivação dos alunos para essas subescalas entre os métodos de ensino Arduino e Tradicional.

Os resultados obtidos para a subescala percepção de escolha da motivação, corroboram com estudos anteriores sobre o uso da Robótica Educacional como técnica de ensino-aprendizagem lúdica promovendo o aumento da motivação e melhoria do aprendizado nas disciplinas introdutórias de programação [Cardoso e Antonello 2015, Stefanello et al. 2013]. Além disso, acredita-se que os tópicos abordados (estrutura de decisão, estrutura de repetição, vetor/matriz) contribuíram para a percepção positiva dos alunos que utilizaram o método Arduino, dado que esses tópicos fazem parte dos conteúdos iniciais de uma linguagem de programação.

\section{Conclusão}

No intuito de analisar a motivação dos alunos sobre o uso da Robótica Educacional como método principal de ensino nas aulas práticas da disciplina de Programação, esse trabalho realizou um estudo experimental norteado pela seguinte questão de pesquisa: "A motivação dos alunos ao utilizar o método Arduino nas aulas práticas da disciplina de Programação é maior do que utilizando o método Tradicional?".

Os resultados, obtidos a partir da aplicação do questionário de motivação IMI, mostraram que o método Arduino teve melhor aceitação dos alunos (percepção de escolha) do que o método Tradicional. Com base nesses resultados, a resposta à questão de pesquisa formulada para este trabalho é positiva, ou seja, a motivação dos alunos 
VIII Congresso Brasileiro de Informática na Educação (CBIE 2019)

Anais do XXV Workshop de Informática na Escola (WIE 2019)

referente a subescala de percepção de escolha foi maior na turma que utilizou o método Arduino (T2) do que na turma que utilizou o método Tradicional (T1).

Espera-se com esse trabalho contribuir para a ampliação das pesquisas na área de Robótica Educacional no âmbito do ensino superior mostrando, por meio do questionário de motivação IMI, a influência positiva do Arduino quando utilizado como método principal de ensino nas aulas práticas de programação. Como trabalho futuro, sugere-se a aplicação desses métodos de ensino em conceitos mais avançados da disciplina de Programação.

\section{Referências}

Banzi, M., Michael S. (2014). Getting started with Arduino: the open source electronics prototyping platform. Make Books - Imprint of: O'Reilly Media, Sebastopol, CA.

Bosse, Y., Gerosa, M. A. (2015). "Reprovações e Trancamentos nas Disciplinas de Introdução à Programação da Universidade de São Paulo: Um Estudo Preliminar”. XXIII Workshop sobre Educação em Computação, Recife, PE.

Cardoso, R., Antonello, S. L. (2015). "Interdisciplinaridade, programação visual e robótica educacional: relato de experiência sobre o ensino inicial de programação". Anais dos Workshops do IV Congresso Brasileiro de Informática na Educação (CBIE), p. 1255-1262.

Deci, E. L., Ryan, R. M. (1985). Intrinsic motivation and self-determination in human behavior. Springer US, $372 \mathrm{p}$.

Freitas, M. F., Mota, S., D. S., Soares, L. S., Reis, R. C. D. (2014). "PORTEC: uma ferramenta para auxiliar na abstração dos conceitos de Estrutura de Dados", XXV Simpósio Brasileiro de Informática na Educação (SBIE), Dourados, MS, p. 872-881.

Pedro, L. Z. (2016). "Uso de Gamificação em Ambientes Virtuais de Aprendizagem para Reduzir o Problema da Externalização de Comportamentos Indesejáveis". Dissertação de Mestrado, Universidade de São Paulo, São Carlos, SP, 152 p.

Piva Jr., D.; Freitas, R. L. (2010). Estratégias para melhorar os processos de Abstração na disciplina de Algoritmos. XXI Simpósio Brasileiro de Informática na Educação (SBIE), João Pessoa - PB, Brasil.

Queiroz, M. O., Rebouças, A. D. D. S. (2018). "Neurociência e o ensino de programação: Uma revisão sistemática da literatura". XXIX Simpósio Brasileiro de Informática na Educação (SBIE), Fortaleza - CE, Brasil, p. 1033-1042.

Santos, F. E. dos, Pereira, D. S., Godin, J. M., Lima, J. V. de, Zaro, M. A.; Filho, A. B. do C. (2018). A Robótica Educativa no Ensino de Lógica de Programação: uma revisão sistemática da literatura. Novas Tecnologias na Educação, v. 16(1): p. 1-10.

Stefanello, D., Filho, J. A. B. L., Guimarães, I. A. S., Almeida, W. R. M. (2013). “A Contribuição Cognitiva da Robótica Educacional como Ferramenta Interdisciplinar no Contexto do Ensino Superior", $19^{\circ}$ Congresso Internacional ABED de Educação a Distância (CIAED), Salvador, Bahia, p.1-10. 